\title{
Chaos and crises in more than two dimensions
}

\author{
Pablo Moresco ${ }^{1}$ and Silvina Ponce Dawson ${ }^{1,2, *}$ \\ ${ }^{1}$ Departamento de Física, Facultad de Ciencias Exactas y Naturales, U.B.A., Ciudad Universitaria, \\ Pabellón I, 1428 Buenos Aires, Argentina \\ ${ }^{2}$ Instituto de Astronomía y Física del Espacio (CONICET), Casilla de Correo 67, Socursal 28,1428 Buenos Aires, Argentina
}

(Received 23 December 1996)

\begin{abstract}
Noisy chaotic trajectories, with finite-time Lyapunov exponents that fluctuate about zero, are basically unshadowable [S. Dawson, C. Grebogi, T. Sauer, and J. A. Yorke, Phys. Rev. Lett 73, 1927 (1994)]. This can occur when periodic orbits, with different numbers of unstable directions, coexist inside the attractor. The presence of a Hénon-type chaotic saddle (i.e., a nonattracting chaotic set with a structure similar to that of the Hénon attractor) guarantees such coexistence in a persistent manner [S. P. Dawson, Phys. Rev. Lett. 76, 4348 (1996)]. In this paper, we describe how these sets appear naturally in maps of more than two dimensions, how they can be found, and what crises they produce. [S1063-651X(97)13005-7]

PACS number(s): 05.45.+b, 05.40.+j
\end{abstract}

\section{INTRODUCTION}

The studies of chaos in low-dimensional dynamical systems have grown steadily during the past decades. In most physical problems, the evolution of a (low-dimensional) dynamical system is given by a set of ordinary differential equations (ODE's) for the variables that define the state of the system. For certain purposes, looking at discrete times provides the necessary information about the system. In these cases the evolution is described by, usually nonlinear, maps. Given a set of ODE's, this can always be achieved provided that a suitable Poincaré surface of section can be found, in which case the corresponding map is called a Poincaré map (see, e.g., [3], p. 64). In this paper, we work with maps which contract the phase space volume, i.e., they are dissipative. We introduce the basic definitions related to maps as dynamical systems in the next section. However, we want to describe, first, the main ideas of the present work. We refer the reader to the next section in case there is some concept whose meaning is not very well known.

In the case of dissipative systems, one is usually interested in the asymptotic behavior, in particular, in what can be observed after a transient disappears. For this reason, most studies of chaotic dynamics have concentrated on chaotic attractors. Chaotic attractors always coexist with nonattracting invariant sets. Moreover, in most cases, these nonattracting sets are tightly related to what can be observed. For example, chaotic attractors contain a dense set of unstable (i.e., "unobservable") periodic orbits [4]. These periodic orbits can be used to calculate the Lyapunov exponents of a typical chaotic trajectory on the attractor [5]. The number of their expanding directions usually determines the number of positive exponents. This means that the sensitivity to initial conditions, which is typical of chaos and is measured by the Lyapunov exponents, is related to properties of the unobservable periodic orbits. Moreover, in the case of flows, the way in which the (unstable) periodic orbits are linked can imply that the attractor is chaotic (see, e.g., [3], p.

*Electronic address: silvina@iafe.uba.ar
222). Other types of phenomena, in which nonattracting invariant sets play an important role, are crises [6]. Crises occur when a chaotic attractor collides with an unstable periodic orbit, in which case, the attractor either changes size suddenly or disappears. Nonattracting sets can themselves be chaotic. Nonattracting chaotic sets also affect the observable dynamics [7]. For example, they can generate transient chaos [8], i.e., trajectories that look chaotic for a while but finally settle down into a periodic orbit. The length of these chaotic transients can increase dramatically with the number of dimensions, showing the necessity of studying chaotic saddles in systems with many degrees of freedom. On the other hand, the structure of both periodic [9] and chaotic [10] nonattracting sets has been determined in real experiments, proving that they leave their imprint on the observable dynamics. This shows that the study of nonattracting sets is not merely academic but it can be of important practical use.

In this paper, we are mainly interested in nonattracting sets that are chaotic, in particular, in the so-called chaotic saddles. The most famous example of this type is the invariant set of the (Smale) horseshoe map [11], which has been "a motivating example for the development of the modern theory of dynamical systems" ( [12], p. 230). The horseshoe map is a $2 \mathrm{D}$ map from the unit square to $R^{2}$. Its invariant set belongs to the unit square. It is at the intersection of two Cantor sets, one of vertical lines and the other of horizontal lines [see Fig. 1(a)]. The invariant set is a chaotic saddle. It contains a dense set of periodic orbits, with one expanding and one contracting direction, and has a Cantor set structure along both the stable (contracting) and the unstable (expanding) directions. As described later, this type of structure is to be expected in the case of 2D dissipative maps [2]. However, as we show in this paper, other types of nonattracting chaotic sets can exist in maps of slightly higher dimension, maps that have not been studied in great detail in the physics literature.

In fact, the maps that have been most widely studied are one-dimensional (noninvertible maps of the interval or maps of the circle) and 2D maps (usually diffeomorphisms) [13]. Chaos can occur in noninvertible $m$-dimensional maps, with $m \geqslant 1$, and in $m$-dimensional diffeomorphisms, with $m \geqslant 2$. Among the dissipative systems, the Hénon family 

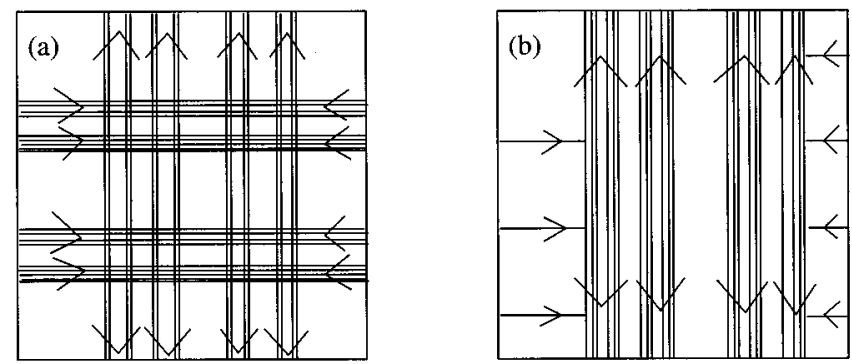

FIG. 1. Schematic picture of a region of a chaotic invariant set that is nonattracting (a) and of one that is attracting (b) for a 2D diffeomorphism of the square to itself. In both pictures the horizontal direction is contracting and the vertical one is expanding. The invariant set in (a) is at the intersection of a Cantor set of vertical lines and a Cantor set of horizontal lines, some of which are shown in the picture. The region shown of the attractor in (b) is a Cantor set of vertical lines. Thus, the invariant set in (a) has a Cantor set structure along the unstable direction while the one in (b) is smooth. The horizontal lines which contain points that approach points in the invariant sets form a Cantor set in (a) while they foliate the whole region shown in (b). For this reason an initial condition randomly chosen in the region shown will approach the invariant set in (b) but not in (a). This shows the difference between the attractor (b) and the nonattracting set in this case.

$$
\begin{gathered}
x_{n+1}=\rho-x_{n}^{2}+b y_{n}, \\
y_{n+1}=x_{n},
\end{gathered}
$$

provides a prototypical example of what can be observed in 2D diffeomorphisms. Changing the values of the two parameters, $\rho$ and $b$, one can find stable periodic orbits, chaotic saddles, and strange attractors on which the dynamics is chaotic. In the case of 2D dissipative diffeomorphisms, and, in particular, for the Hénon map, these chaotic saddles have the same structure as the invariant set of the Smale horseshoe, i.e., they are products of Cantor sets. On the other hand, the chaotic attractors of this map also contain a dense set of periodic orbits with one expanding and one contracting direction, but they are "smooth" along the unstable (expanding) directions [15]. The "smoothness" along the unstable directions is the basic difference between a chaotic attractor and a chaotic saddle in 2D, and it is related to what is called the absolute continuity of the stable foliation [17] (p. 216). We show this difference, schematically, in Fig. 1, where we depict parts of two chaotic invariant sets of a 2D diffeomorphism. The invariant set, $\Lambda$, in Fig. 1(a) is nonattracting (of horseshoe type). It is the intersection of a Cantor set of vertical lines and a Cantor set of horizontal lines, some of which are shown in the figure. The invariant set, $\mathcal{A}$, in Fig. 1(b) is an attractor (of Hénon type). The piece we show is a Cantor set of vertical lines. All the region shown in Fig. 1(b) is "foliated" by the stable sets of the points in $\mathcal{A}$ (i.e., by points that approach $\mathcal{A}$ under forward iterations of the map). On the other hand, the stable set of $\Lambda$ is a Cantor set of horizontal lines. While there is a nonvanishing probability that, by choosing an initial condition at random in the region shown in Fig. 1(b), we will end up approaching $\mathcal{A}$, there is a zero probability that an analogous thing will happen with $\Lambda$ in Fig. 1(a), since we would need to lie exactly on one of

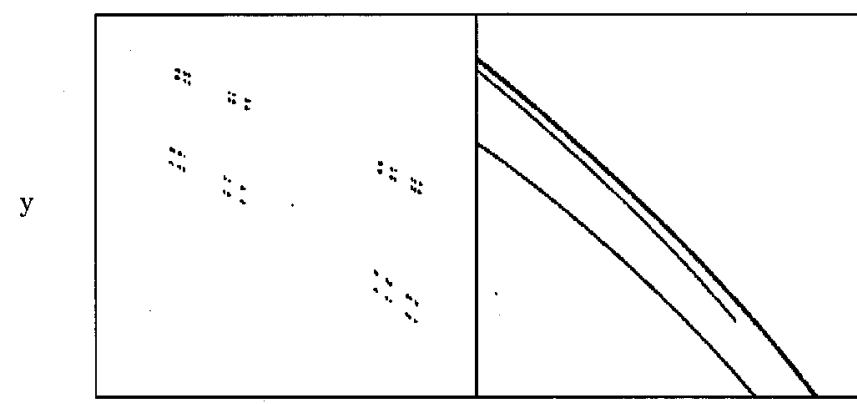

$\mathrm{x}$

FIG. 2. An actual realization of the scheme shown in the previous figure that occurs for the Hénon map [Eq. (1)] with $b=0.3$. The figure on the left corresponds to the piece of a chaotic saddle at $\rho=4.2$ contained in $1 \leqslant x \leqslant 3,0.7 \leqslant y \leqslant 2$. The figure on the right is the piece of an attractor at $\rho=1.2$ contained in $0.7 \leqslant x \leqslant 1.3$, $0.5 \leqslant y \leqslant 0.9$. These figures were made using the software DYNAMICS as described in [18].

the horizontal lines of the stable foliation. For this reason, $\Lambda$ is nonattracting while $\mathcal{A}$ is an attractor. We also show pictures of a chaotic saddle (of horseshoe type) and a chaotic attractor of the Hénon map in Fig. 2, where the different type of structures can be observed.

In higher-dimensional maps, other types of nonattracting chaotic sets can exist, in particular, chaotic saddles that are smooth along some unstable directions [2]. In almost all the examples of maps that we can think of, there are invariant manifolds (i.e., manifolds, $M$, such that if we pick up an initial condition in $M$ all forward and backward iterations of it stay in $M$ ), which are of lower dimension than the whole phase space. Typical examples are the stable manifolds of unstable periodic orbits (see the next section). The chaotic saddles that we describe in this paper belong to an invariant manifold of lower dimension than the phase space. Moreover, they are chaotic attractors with a structure similar to the Hénon attractor, if we restrict the dynamics to the invariant manifold. However, when we look at the whole phase space, they are not attractors, because the manifold itself is not attracting. We call these sets Hénon saddles. In [2] we described how these Hénon saddles could arise via a sequence of bifurcations out of unstable periodic orbits. For this reason we expected them to appear as often as strange attractors in maps of more than 2D. In fact, we show in this paper several examples of Hénon saddles that are formed in this way. All the examples correspond to a 4D map that models a particular physical system, the double-rotor map $[19,20]$ (see Sec. III), which has not been tailored to display this behavior. Furthermore, it has been proved that, under certain conditions, homoclinic bifurcations (associated with orbits homoclinic to fixed points with at least two unstable directions) give rise to the formation of Hénon saddles [21], in very much the same way as Hénon attractors appear in homoclinic bifurcations of 2D diffeomorphisms [22]. Moreover, the result in [22] states that these Hénon attractors can be found, with a nonvanishing probability, by choosing the parameters at random. All these results reinforce our idea that Hénon saddles should be found very often in maps of more than 2D.

As described in [2], the existence of a Hénon saddle can give rise to (persistent) trajectories that visit the vicinity of 
periodic orbits with different numbers of unstable directions. In fact, we describe in detail an example, in Sec. IV, in which a chaotic saddle of horseshoe type, containing periodic orbits with 1D unstable manifolds, "collides", with a Hénon saddle, giving rise to an invariant set that contains both $1 \mathrm{D}$ and 2D unstable periodic orbits. Again, this example corresponds to the double-rotor map, a map that describes the dynamics of a simple mechanical system. Moreover, in this example, if we change the parameters, a crisis occurs, after which the invariant set, with its $1 \mathrm{D}$ and 2D unstable orbits, belongs to the attractor of the system. This type of behavior can occur in a persistent way because the set of points that approach the Hénon saddle (its stable set) is of larger dimension than the stable manifolds of its periodic points. As described in Sec. III, this property also allows us to find, numerically, Hénon saddles that contain 2D unstable periodic orbits (i.e., with two expanding directions) using a technique [23] that was developed for chaotic saddles (of horseshoe type) with only 1D unstable periodic orbits.

A trajectory that visits the vicinity of periodic orbits with different numbers of expanding directions will have at least one finite-time Lyapunov exponent that changes sign along its way. If this causes the exponent to fluctuate about zero, then any noise added to the trajectory (such as the one that is unavoidable in numerical simulations) makes the noisy trajectory continuously unshadowable [1]. This means that there is no continuous deformation of the noisy trajectory that can reduce the noise all the way down to zero. Thus, numerically generated trajectories of this type are highly unreliable. On the other hand, if the noise is intrinsic, in the sense that is produced by variables that are left aside during the modeling, this problem raises the concern of how valid the model itself is. Although some years ago there was some debate around this question [24], we think it still remains an open problem. Fluctuating Lyapunov exponents are also associated to other dramatic behaviors, such as riddled basins, blow-out bifurcations, and on-off intermittency [14].

The organization of the paper is as follows. In Sec. II, we introduce some basic definitions providing an intuitive picture of what they mean. In Sec. III, we describe how Hénon saddles arise via a sequence of bifurcations out of unstable periodic orbits, showing several examples in which this is realized. In Sec. IV, we comment on the crises that can occur when there is a Hénon saddle. In particular, we describe, in detail, the example of a chaotic saddle of the double-rotor map that collides with a Hénon saddle. We analyze, in this case, how the various finite-time Lyapunov exponents behave. In Sec. V, the conclusions are summarized.

\section{BASIC DEFINITIONS}

In this paper, a dynamical system is a map, $f: \mathcal{M} \rightarrow \mathcal{M}$, from a phase space, $\mathcal{M}$, to itself. The phase space is an $m$-dimensional $(m \mathrm{D})$ differentiable manifold such that a point in it corresponds to one state of the real system [25]. For the sake of simplicity, we will consider $\mathcal{M} \subseteq R^{m}$. Let us assume that $f$ is a diffeomorphism (as it is always the case for Poincare maps of smooth flows). In such a case, an orbit is a sequence $\left\{x_{n}\right\}_{n \in Z}$ such that $x_{n+1}=f\left(x_{n}\right)$. The forward orbit is the subsequence with $n \geqslant 0$. A subset of phase space, $A \subset \mathcal{M}$, is called invariant if it is mapped onto itself as the system evolves forward or backwards in time, i.e., $f^{n}(A) \subseteq A$ for $n \in Z$, where we denote by $f^{n}$ the $n$-fold composition of $f$ with itself, if $n \geqslant 0$ and that of the inverse, $f^{-1}$, if $n<0 . A \subset \mathcal{M}$ is positively invariant if $f^{n}(A) \subseteq A$ for $n \in Z, n \geqslant 0$.

The simplest examples of invariant sets are the fixed points [points $\bar{x} \in \mathcal{M}$ such that $f(\bar{x})=\bar{x}$ ] and the periodic points [points $\bar{x} \in \mathcal{M}$ such that $f^{p}(\bar{x})=\bar{x}$ with $p \in N$ ]. An orbit that starts at such a periodic point is periodic of period $p$. Fixed points are periodic orbits of period 1. The dynamics in the vicinity of a fixed point or periodic orbit can usually be analyzed by linearizing the map $f^{p}$ around the corresponding periodic point, $\bar{x}$. In this way the (local) dynamics is given by $y_{n+1}=D f^{p}(\bar{x}) y_{n}$, where $y_{n}=x_{n}-\bar{x}$ and $D f^{p}(\bar{x})$ is the derivative matrix of $f^{p}$ at the periodic point, $\bar{x}$. According to the Hartman-Grobman theorem [12], the linearization provides meaningful information if the periodic point is hyperbolic, i.e., if the norms of all the eigenvalues of $D f^{p}(\bar{x})$ are different from 1 . The subspace spanned by the (generalized) eigenvectors of $D f^{p}(\bar{x})$ with norm less than 1 is called the stable subspace, $E^{s}(\bar{x})$, and the one spanned by those with norm bigger than 1 is called the unstable subspace, $E^{u}(\bar{x})$. If all the eigenvalues of $D f^{p}(\bar{x})$ have norm less than 1, then, the periodic point is stable [26]. Moreover, it is asymptotically stable, i.e., there is a neighborhood $U_{\bar{x}}$ of $\bar{x}$ such that if $x \in U_{\bar{x}}$, then $f^{p n}(x) \rightarrow \bar{x}$ as $n \rightarrow \infty$. On the other hand, if there is at least one eigenvalue with norm bigger than 1 , then the fixed point is unstable.

Given a periodic point, $\bar{x}$, of a diffeomorphism, $f$, there are also global stable and unstable manifolds, $W^{s}(\bar{x})$ and $W^{u}(\bar{x})$, which are as smooth as $f$, have the same dimension as the subspaces $E^{s}(\bar{x})$ and $E^{u}(\bar{x})$, respectively, and are such that $W^{s}(\bar{x})\left[W^{u}(\bar{x})\right]$ is tangent to $E^{s}(\bar{x})\left[E^{u}(\bar{x})\right]$ at $\bar{x}$. If $\bar{x}$ is hyperbolic, then, $\operatorname{dim}\left(W^{s}(\bar{x})\right)+\operatorname{dim}\left(W^{u}(\bar{x})\right)=\operatorname{dim}(\mathcal{M})$, while $W^{s}(\bar{x})=\left\{x: f^{p n}(x) \rightarrow \bar{x} \quad\right.$ as $\left.n \rightarrow \infty\right\}$ and $W^{u}(\bar{x})$ $=\left\{x: f^{p n}(x) \rightarrow \bar{x}\right.$ as $\left.n \rightarrow-\infty\right\}$. In this case, the stable and unstable manifolds are the set of all points that tend to the periodic point, $\bar{x}$, under forward or backward iterations of $f^{p}$, respectively. These manifolds are invariant sets whose organization in phase space provides information about the dynamics of the system. In particular, if there is a hyperbolic fixed point, $\bar{x}$, and we pick up an initial condition sufficiently close to $W^{s}(\bar{x})$, the trajectory will approach $\bar{x}$ for a while until it will eventually move to some other place, "following,' perhaps, other manifolds. The stable and unstable manifolds of a periodic point, $\bar{x}$, can also intersect at points other than $\bar{x}$, which are called homoclinic points. A homoclinic point, $q$, is such that $f^{p n}(q) \rightarrow \bar{x}$ as $n \rightarrow \pm \infty$. The corresponding orbit is called a homoclinic orbit. The existence of homoclinic orbits gives rise to very complicated dynamics. In particular, they imply the existence of invariant sets similar to those of the Smale horseshoe (see, e.g., [12], p. 252). Given two fixed (or periodic) points, $\overline{x_{1}}$ and $\overline{x_{2}}$, there can be points $q \in W^{s}\left(\overline{x_{1}}\right) \cap W^{u}\left(\overline{x_{2}}\right)$, or $q \in W^{u}\left(\overline{x_{1}}\right)$ $\cap W^{s}\left(\overline{x_{2}}\right)$, which are called heteroclinic, and are also associ ated to complicated dynamics.

In the preceding section, we talked about attractors. An attractor is the invariant set on which the observable asymptotic evolution "occurs.', More precisely, an attractor is a closed invariant set, $\mathcal{A} \in \mathcal{M}$, with a dense orbit and with 
the property that it has an open neighborhood, $U \supset \mathcal{A}$, which contains a subset, $U_{+}\left(U_{+} \subset U\right)$, of positive Lebesgue measure, such that, if $x \in U_{+}$, then $f^{n}(x) \rightarrow A$ as $n \rightarrow \infty$ [12]. This means that, if one chooses an initial condition at random in $U$, then there is a nonvanishing probability that its forward orbit will approach the attractor [as in the case of Fig. 1(b), for example]. Numerically generated pictures of attractors are based on this fact: an initial condition is picked up at random and points of the forward orbit, with $n$ big enough, are drawn. Provided that this is done with no errors, one obtains a picture which, to the eye, is indistinguishable from the attractor. The set of points whose forward orbits approach the attractor is called the basin of attraction.

Asymptotically stable fixed points or periodic orbits are examples of attractors. As already mentioned, attractors can also be strange and the dynamics on them can be chaotic. A chaotic attractor is an attractor that has a dense set of (unstable) periodic points and exhibits sensitive dependence on initial conditions [4]. This means that two initial conditions, which are very close to one another, generate forward orbits that diverge (exponentially) from one another. One way of characterizing this sensitivity is by means of the Lyapunov exponents. These numbers quantify the average rate of expansion and contraction along the different directions in phase space, as the system evolves. Lyapunov exponents only exist for points $x \in \mathcal{M}$ which are called regular [27]. A point $x \in \mathcal{M}$ is regular if there are numbers $\lambda_{1}(x)>\lambda_{2}(x)>\cdots>\lambda_{s}(x)$ and a decomposition of the tangent space of $\mathcal{M}$ at $x, T_{x} \mathcal{M}$, of the form $T_{x} \mathcal{M}=E_{1}(x)$ $\oplus \cdots \oplus E_{s}(x)$, such that

$$
\lim _{n \rightarrow \infty} \frac{1}{n} \log \left\|\left(D f^{n}(x)\right) u\right\|=\lambda_{j}(x),
$$

for every $u \in E_{j}(x), u \neq 0$ and every $1 \leqslant j \leqslant s$ [27]. The numbers, $\lambda_{j}(x)$, are the Lyapunov exponents and they are unique. The decomposition in the eigenspaces $E_{j}(x)$ is also unique. We call a finite-time (or time-T) Lyapunov exponent the number $\lambda_{j}$, obtained as in Eq. (2), but for $n$ finite $(n=T)$. A result due to Oseledec [28] states that almost every point in an attractor is regular. Moreover, all "typical points" in a given attractor have the same Lyapunov exponents [29]. For this reason one associates the Lyapunov exponents with the attractor. A positive Lyapunov exponent means that there is an expanding direction, and therefore, sensitivity to initial conditions and chaos. The value of a positive Lyapunov exponent provides an indication of how much two nearby orbits separate in time. Chaotic saddles are invariant sets which have a dense set of periodic orbits with at least 1D stable manifolds, and display sensitive dependence on initial conditions, i.e., they have a positive Lyapunov exponent.

Finally, we will call a Hénon attractor any chaotic attractor such that all its periodic orbits are 1D unstable, and that is smooth along the unstable manifolds [i.e., that is similar to the chaotic attractors found for the Hénon family (1)]. We will call a Hénon saddle a chaotic saddle that belongs to an invariant manifold of lower dimension than the phase space and such that, on this manifold, it is a Hénon attractor. While a typical trajectory on a Hénon attractor has only one positive Lyapunov exponent, one on a Hénon saddle has at least
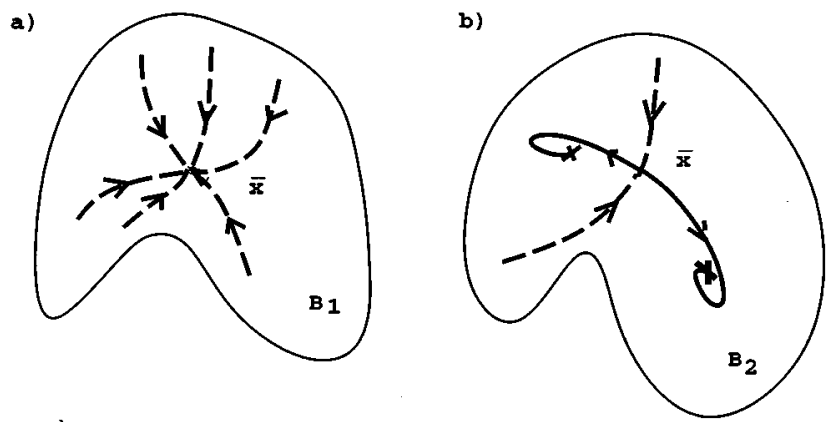

c)

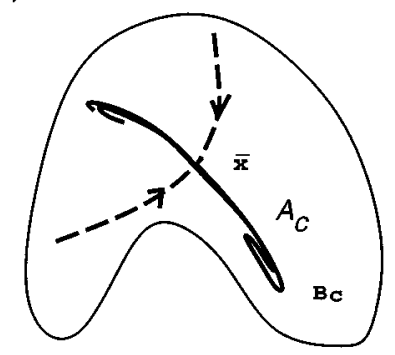

FIG. 3. A schematic picture of how a Hénon attractor can arise via a sequence of bifurcations. At $\rho=\rho_{1}$ the attractor is a stable fixed point whose stable manifold is the basin of attraction, $B_{1}$ (a). At $\rho=\rho_{1}^{*}, \rho_{1}<\rho_{1}^{*}<\rho_{2}, \bar{x}$ suffers a period doubling bifurcation and a period 2 orbit is born. At $\rho=\rho_{2}$ the period 2 orbit is stable (it is the new attractor) while $\bar{x}$ is unstable. We show its 1D unstable manifold (b). The sequence of bifurcations goes on, until at $\rho=\rho_{c}$ the attractor is chaotic, contains all the periodic orbits that were involved in the sequence of period doubling bifurcations, which at $\rho=\rho_{c}$ are 1D unstable, and is smooth along the unstable manifolds (c). At least part of the basin of attraction, $B_{c}$, at $\rho=\rho_{c}$, comes from $B_{1}$ by a smooth deformation.

two. In the latter case, the eigenspaces, $E_{j}$, associated to one of the positive Lyapunov exponents are always tangent to the invariant manifold, $\mathcal{N}$, on which the saddle is attracting, while those associated to the other positive exponents are transverse to it. All the examples we show in this paper have only two positive Lyapunov exponents, one with its eigenspace tangent to $\mathcal{N}$ and the other one with its eigenspace transverse to $\mathcal{N}$.

\section{FROM BIFURCATIONS OF PERIODIC ORBITS TO HÉNON SADDLES}

Let us recall how a Hénon attractor may appear via a sequence of bifurcations, as a parameter is varied. For this purpose, let us consider an $m \mathrm{D}$ diffeomorphism that depends on a parameter, $\rho$. For some value of $\rho$, e.g., $\rho_{1}$, the attractor, $\mathcal{A}_{1}$, is a stable fixed (or periodic) point, $\bar{x}$. At $\rho=\rho_{1}$, the $m \mathrm{D}$ stable manifold of $\bar{x}$ is the basin of attraction of the attractor [see Fig. 3(a)]. Let us call this basin $B_{1}$. At $\rho=\rho_{1}^{*}$, the fixed point undergoes a period doubling bifurcation and becomes (1D) unstable. Immediately after the bifurcation, at $\rho=\rho_{2}>\rho_{1}^{*}, \bar{x}$ has a 1D unstable and an $(m-1)$-dimensional stable manifold. Meanwhile, the new attractor, $\mathcal{A}_{2}$, is a period 2 orbit whose basin of attraction, $B_{2}$, is such that, at least part of it, is a smooth deformation of $B_{1}$ [30] [see Fig. 3(b)]. At some other parameter value, $\rho=\rho_{2}^{*}$, the period 2 orbit becomes 1D unstable and the new 


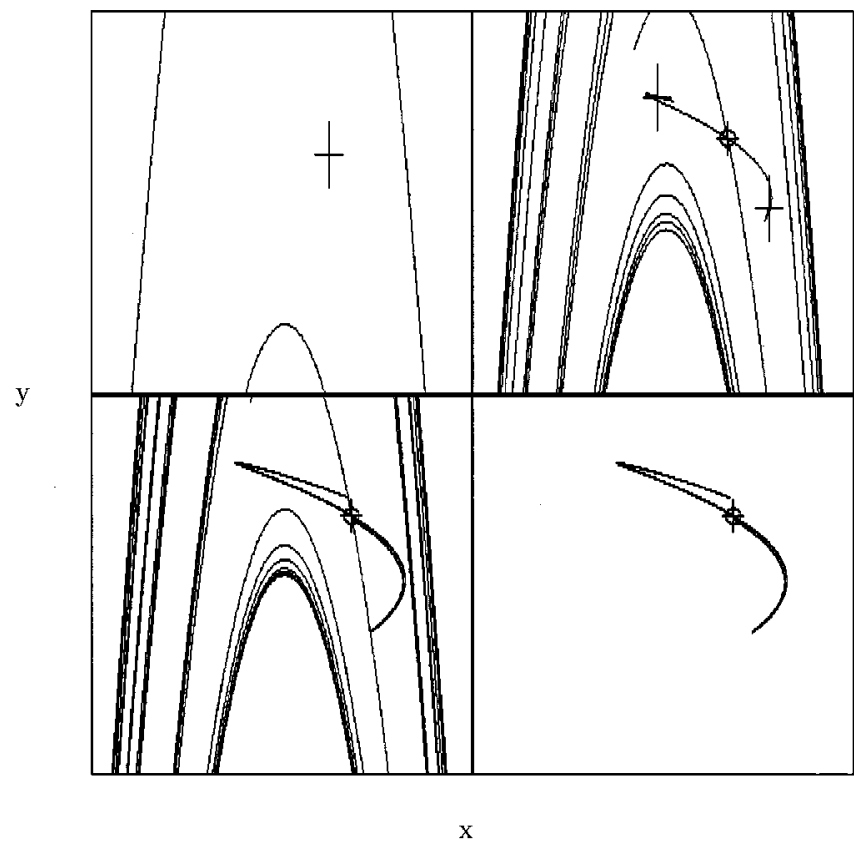

FIG. 4. An actual realization of the scheme described in the previous figure that occurs in the Hénon map [Eq. (1)] with $b=-0.3$. The figure on the upper left shows the attractor at $\rho=1.2$ and (part of) the basin boundary. The attractor is a fixed point indicated with a cross. The figure on the upper right corresponds to $\rho=1.8$. At this value the attractor is a period 2 orbit (shown with crosses). Also shown is the period 1 orbit which was attracting at $\rho=1.2$ (indicated with a cross surrounded by a circle), its unstable and part of its stable manifold. The unstable manifold has two branches, each of which approaches a point of the period 2 orbit. As before, part of the attractor's basin boundary is also drawn. The figure on the lower-left corresponds to $\rho=2.02$. Here we show the same period 1 orbit, its stable and unstable manifolds and part of the basin boundary. The attractor in this case is chaotic (a Hénon attractor). It is shown in the figure on the lower-right. We can see that it is indistinguishable from the unstable manifold of the period 1 orbit. In all figures $-2.5 \leqslant x \leqslant 2.5$ and $-2.5 \leqslant y \leqslant 2.5$.

attractor is a period 4 orbit instead. The new basin, $B_{3}$, is such that, at least part of it, is a smooth deformation of $B_{2}$. This picture goes on until, eventually, we have a chaotic attractor, $\mathcal{A}_{c}$ [see Fig. 3(c)]. We do not want to describe everything that happens for all parameter values, but at some value, $\rho=\rho_{c}$, there is a Hénon attractor that contains all the 1D unstable periodic orbits that suffered the period-doubling bifurcations just described. This attractor is the closure of the unstable manifold of $\bar{x}[16]$ and it has one positive Lyapunov exponent. As before, at least part of the basin of the Hénon attractor, $B_{c}$, "comes from" a smooth deformation of $B_{1}$. We show a schematic picture of this process in Fig. 3, and an actual realization, for the Hénon map, in Fig. 4.

Let us consider, now, a similar picture, but one in which the primordial fixed point, $\bar{x}$, is 1D unstable (and hyperbolic) from the very beginning. Therefore, the stable manifold of $\bar{x}$ at $\rho=\rho_{1}$ is an $(m-1)$-dimensional invariant manifold. Let us call this manifold $B_{1}$. On $B_{1}$, the fixed point, $\bar{x}$, is attracting. Let us suppose that, at $\rho=\rho_{1}^{*}, \bar{x}$ suffers a perioddoubling bifurcation, immediately after which it becomes $2 \mathrm{D}$ unstable, while a 1D unstable period 2 orbit is born. The

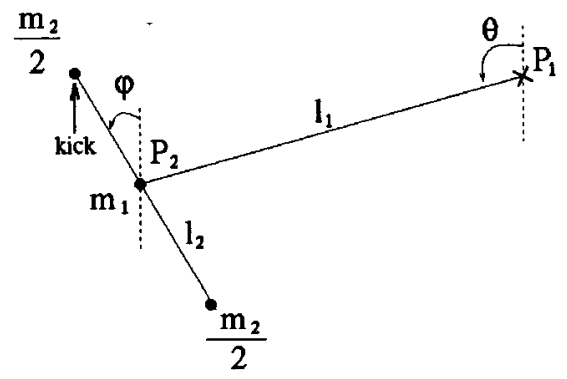

FIG. 5. The physical system that leads to the double-rotor map.

$(m-1)$-dimensional stable manifold of the period 2 orbit, immediately after the bifurcation, is a smooth deformation of $B_{1}$. Basically, the same sequence of pictures as those shown in Fig. 3 hold in this case, but on the $(m-1)$-dimensional invariant manifold that, at $\rho=\rho_{1}$, is the stable manifold of $\bar{x}$. In this case, the invariant set, at $\rho=\rho_{c}$, is a Hénon saddle: a chaotic saddle that is attracting on an invariant manifold of lower dimension than the full phase space, and whose typical trajectories have two, rather than one, positive Lyapunov exponents. This invariant manifold is a "remnant" of the stable manifold of the primordial unstable periodic orbit, and, on this invariant manifold, the Hénon saddle is attracting. In fact, we have found several examples of Hénon saddles that arise in this way, via a sequence of bifurcations out of unstable periodic orbits, in the double-rotor map.

The double-rotor map $[19,20]$ is the return map of a system describing the evolution of two connected rods of lengths $\ell_{1}$ and $\ell_{2}$, moving on a plane under the effect of $\delta$ kicks and damping $[19,20]$. One of the rods rotates about a fixed pivot, while the other one rotates around the other end of the first rod. There are masses, $m_{1}$ and $m_{2}$, at the free ends of both rods, and there is friction at both pivots (see Fig. 5). $\delta$ kicks of amplitude $\rho$ are applied to one end of the second rod periodically, with period $T$. Given this system, it is easy to obtain a map, relating the angles $(x \equiv \theta, y \equiv \varphi)$ and velocities $(z \equiv \dot{\theta}, w \equiv \dot{\varphi})$ immediately after the $(n+1)$ th kick with those immediately after the $n$th one [19]. This map is of the form

$$
\begin{gathered}
x_{n+1}=x_{n}+M_{11} z_{n}+M_{12} w_{n}, \quad \bmod (2 \pi), \\
y_{n+1}=y_{n}+M_{21} z_{n}+M_{22} w_{n}, \quad \bmod (2 \pi), \\
z_{n+1}=\frac{\rho \ell_{1}}{I_{1}} \sin x_{n+1}+L_{11} z_{n}+L_{12} w_{n}, \\
w_{n+1}=\frac{\rho \ell_{2}}{I_{2}} \sin y_{n+1}+L_{21} z_{n}+L_{22} w_{n},
\end{gathered}
$$

where $M_{i j}$ and $L_{i j}$ are elements of constant $2 \times 2$ matrices, $L, M$, which depend on the lengths of the rods, the masses, the period of the kicks, and the friction coefficients at the pivots [20], and $I_{i}$ are the moments of inertia about the pivots. We keep all the parameters fixed at the same values as those used in [20], with the exception of $\rho$, which we vary for different numerical experiments. 

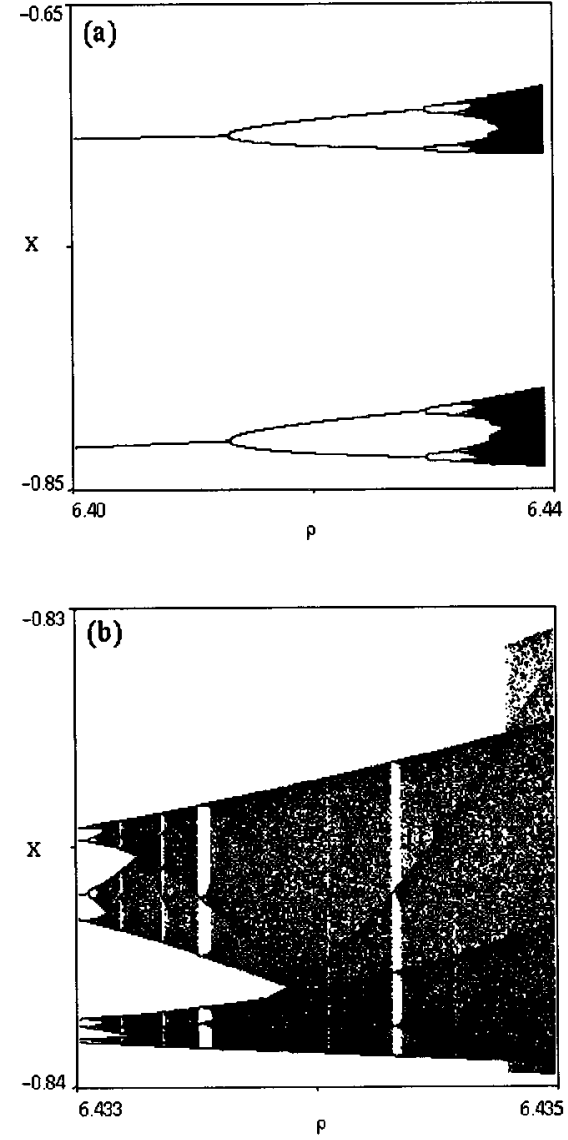

FIG. 6. (a) Bifurcation diagram of invariant sets of the doublerotor map that are nonattracting in the full phase space, but are attracting inside an invariant 3D manifold. All points in the figure have been obtained using the PIM-triple method. (b) Blowup of the figure in (a) in which the structure of windows is evident. Clearly, the diagrams are completely similar to those obtained for attractors.

In [2] we could follow the first steps of a period-doubling cascade out of an unstable periodic orbit of (3). This led us to think that this map was a good candidate for the formation of Hénon saddles. In fact, using the PIM-triple method [23,18], as described in the Appendix, we have been able not only to find Hénon saddles in this map, but also to follow all the sequence of bifurcations out of an unstable periodic orbit that lead to the formation of the saddle. In this way, we could generate bifurcation diagrams of nonattracting invariant sets, as functions of $\rho$, for the double-rotor map. We show an example in Fig. 6.

The invariant sets shown in Fig. 6 are attracting inside an invariant 3D manifold, $\mathcal{N}$ (which also changes with $\rho$ ). The invariant set, at $\rho=6.40$, is a $1 \mathrm{D}$ unstable and hyperbolic period 2 orbit, $\bar{x}$. It is born at a "saddle-node" bifurcation, at $\rho \approx 6.36$. Actually, immediately after this bifurcation, none of the new periodic orbits is stable: one is 1D unstable while the other one is $2 \mathrm{D}$ unstable. The invariant manifolds, $\mathcal{N}(\rho)$, on which the sets shown in Fig. 6 are attracting, come from a smooth deformation of $W^{s}(\bar{x})$. At $\rho=6.44$, there is no longer an invariant set that is attracting in $\mathcal{N}$. The Hénon saddle suffers a crisis at $\rho \approx 6.4395$, when it collides with a 2D unstable periodic orbit. For larger values of $\rho$, a horseshoe-type chaotic saddle, with 2D unstable manifolds,

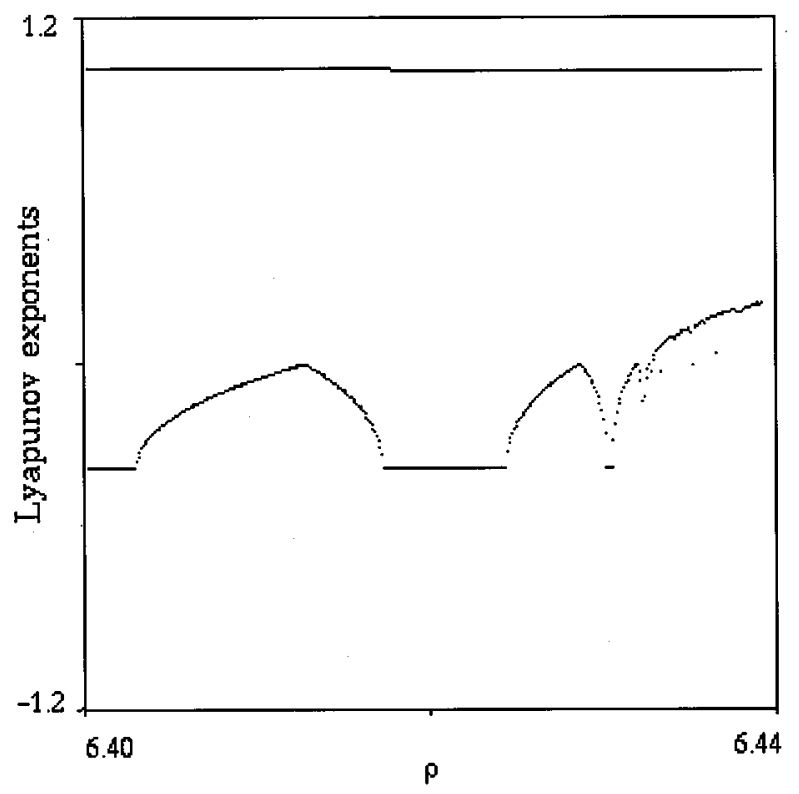

FIG. 7. The two largest Lyapunov exponents associated with the invariant sets shown in Fig. 6(a) as functions of $\rho$. There is always one positive exponent, which means that the sets are nonattracting, and a second one that becomes positive when the invariant set becomes a Hénon saddle.

remains in the region previously occupied by the Hénon saddle. We show in Fig. 7 the two largest Lyapunov exponents associated with the invariant sets of Fig. 6(a). There, we see that there is always one positive exponent and a second one, that becomes positive when the invariant set becomes chaotic. Therefore, the second Lyapunov exponent is associated to the expanding direction that is contained in $\mathcal{N}$, while the first one corresponds to the expansion transverse to this manifold. As explained in the Appendix, the fact that the expansion transverse to $\mathcal{N}$ is stronger than the one along $\mathcal{N}$, for all values of $\rho$, allowed us to obtain this diagram using the PIM-triple method.

We show in Fig. 8 a projection of the Hénon saddle onto the $x-z$ plane at $\rho=6.439$. There, we can observe that the structure is similar to that of a Hénon attractor (compare with Fig. 2). It is smooth along one unstable direction and it has a Cantor set structure along the stable one.

As mentioned before, the PIM-triple method was originally developed for chaotic saddles of horseshoe type with only one unstable direction [23]. However, it is based on a series of assumptions that are fulfilled by certain Hénon saddles with two positive Lyapunov exponents. The method consists, first, of obtaining a point arbitrarily close to the stable set of the saddle. This first part of the method converges under two assumptions. The first assumption is that we can generically intersect the stable set of the saddle with a segment. In an $m$-dimensional phase space, a Hénon saddle with two positive Lyapunov exponents is attracting in an $(m-1)$-dimensional invariant manifold. Therefore, simple dimensional arguments show that a segment can generically intersect such a manifold, and thus, the stable set of the saddle (see the Appendix). Moreover, the fact that we could find a nonattracting chaotic set with two expanding directions by "probing" the space with a segment is, per se, an indication that the stable set of the saddle is of (at least) 


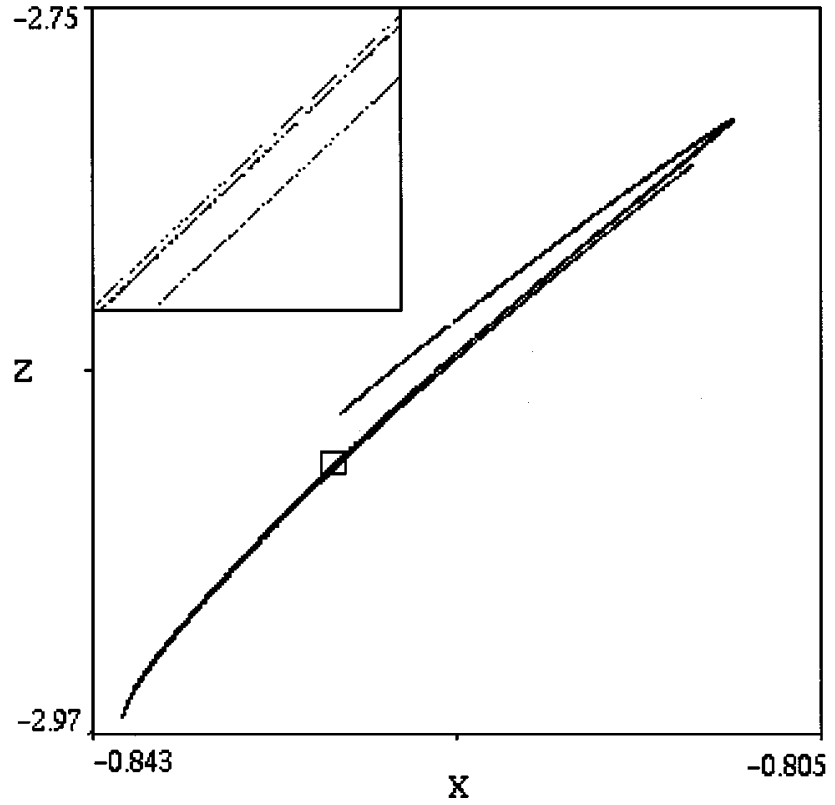

FIG. 8. A plot of $z$ vs $x$ for a trajectory on a Hénon saddle of the double-rotor map with $\rho=6.439$. Inset: a blowup of the region indicated in the figure.

$(m-1)$ dimensions, i.e., a Hénon saddle. The second assumption of the PIM-triple method is that the intersection of the segment and the stable set consists of disconnected points. Although the invariant manifold, and thus the stable set of the saddle, can fold onto itself, there will always be gaps in between foldings, so that the intersection of the manifold with the segment will (generically) be at most a Cantor set. Once a point arbitrarily close to the stable set is found, a chaotic orbit, "almost on' the saddle, is generated. Having an invariant set with two expanding directions introduces new effects in this case, which are not discussed in [23]. As explained in more detail in the Appendix, we will generate such an orbit if the expansion transverse to the invariant manifold, $\mathcal{N}$, is stronger than the one along $\mathcal{N}$. All the assumptions of the PIM-triple method also hold for 1D unstable hyperbolic orbits. This is the reason why we could produce the whole bifurcation diagram of Fig. 6 using this method.

\section{CRISES INVOLVING HÉNON SADDLES IN THE DOUBLE-ROTOR MAP}

As mentioned in the preceding section, a segment can generically intersect the stable set of a Hénon saddle with two positive Lyapunov exponents. Actually, any 1D manifold can do it, since such a saddle is attracting on an $(m-1)$-dimensional manifold (as before, $m$ is the phase space dimension), in particular, the unstable manifold of a 1D unstable periodic orbit (a periodic orbit with one expanding direction). Suppose that the periodic orbit is hyperbolic. Then, its stable manifold has $(m-1)$ dimensions. Therefore, it can be generically intersected by a manifold of one or more dimensions, in particular, the unstable manifold of any periodic orbit in the Hénon saddle. Thus, as described in [2], in such a case, it is possible to have orbits that repeatedly visit the vicinity of periodic points with different numbers of
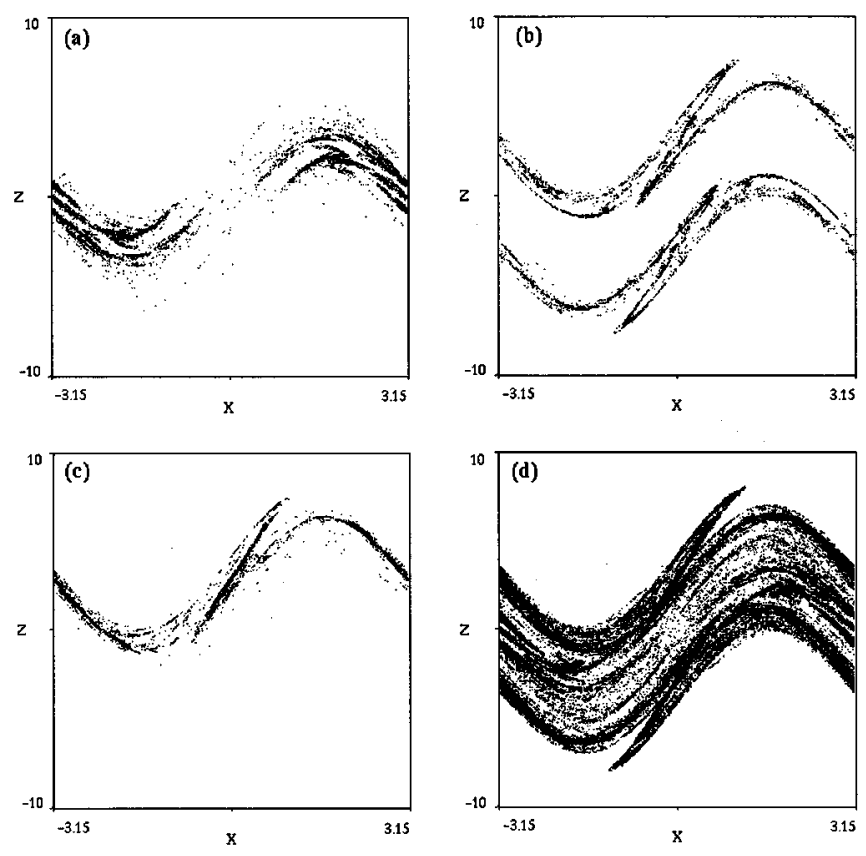

FIG. 9. (a) A projection of a horseshoe saddle of the doublerotor map at $\rho=5.28$. All the periodic orbits that we looked at in this saddle were 1D unstable. (b) Similar to (a) but for two Hénon saddles at $\rho=5.28$. One of them is the set on the top, the other one the set on the bottom. They are related by a symmetry transformation. All the periodic orbits that we looked at in these saddles were 2D unstable. (c) Similar to (a) but for another horseshoe saddle at $\rho=5.40$. There is also another saddle symmetrically conjugated to this one at the bottom, which we have not drawn. (d) A nonattracting invariant set at $\rho=5.49$ that contains both 1D and 2D unstable periodic orbits that seems to contain the saddles in (a), (b), and (c).

unstable directions. Furthermore, it was mentioned in [2] that these orbits could be created as a critical parameter value was approached, at which new intersections between the relevant manifolds were created. In fact, we show in this paper an example of this type of crisis that occurs in the doublerotor map.

The example we will show is a little bit more complicated, since we could identify three nonattracting sets that seem to play a role in the process. We show in Fig. 9(a) a picture of a chaotic saddle of horseshoe type, for the doublerotor map with $\rho=5.28$. The picture is actually a projection of the saddle on the $x-z$ plane, which was obtained using the PIM-triple method. All the periodic orbits in this saddle have 1D unstable manifolds (at least, all the ones that we looked at). For this reason, typical trajectories on the saddle have only one positive Lyapunov exponent. Moreover, the distribution of finite-time Lyapunov exponents is peaked around its mean value, as may be observed in Figs. 10(a) and 10(c). We could follow this chaotic saddle for quite a long interval of parameter values. For $5.28 \leqslant \rho \leqslant 5.47$, it coexists with (at least) two Hénon saddles with two positive Lyapunov exponents, i.e., saddles in which all periodic orbits have two unstable directions. These two Hénon saddles are related by a symmetry transformation. We show a projection of them, at $\rho=5.28$, in Fig. 9(b). The distribution of the first and second finite-time Lyapunov exponents of this saddle are also shown in Figs. 10(a) and 10(c). At $\rho=\rho^{*}$, with 5.47< $\rho^{*}<5.49$, the 

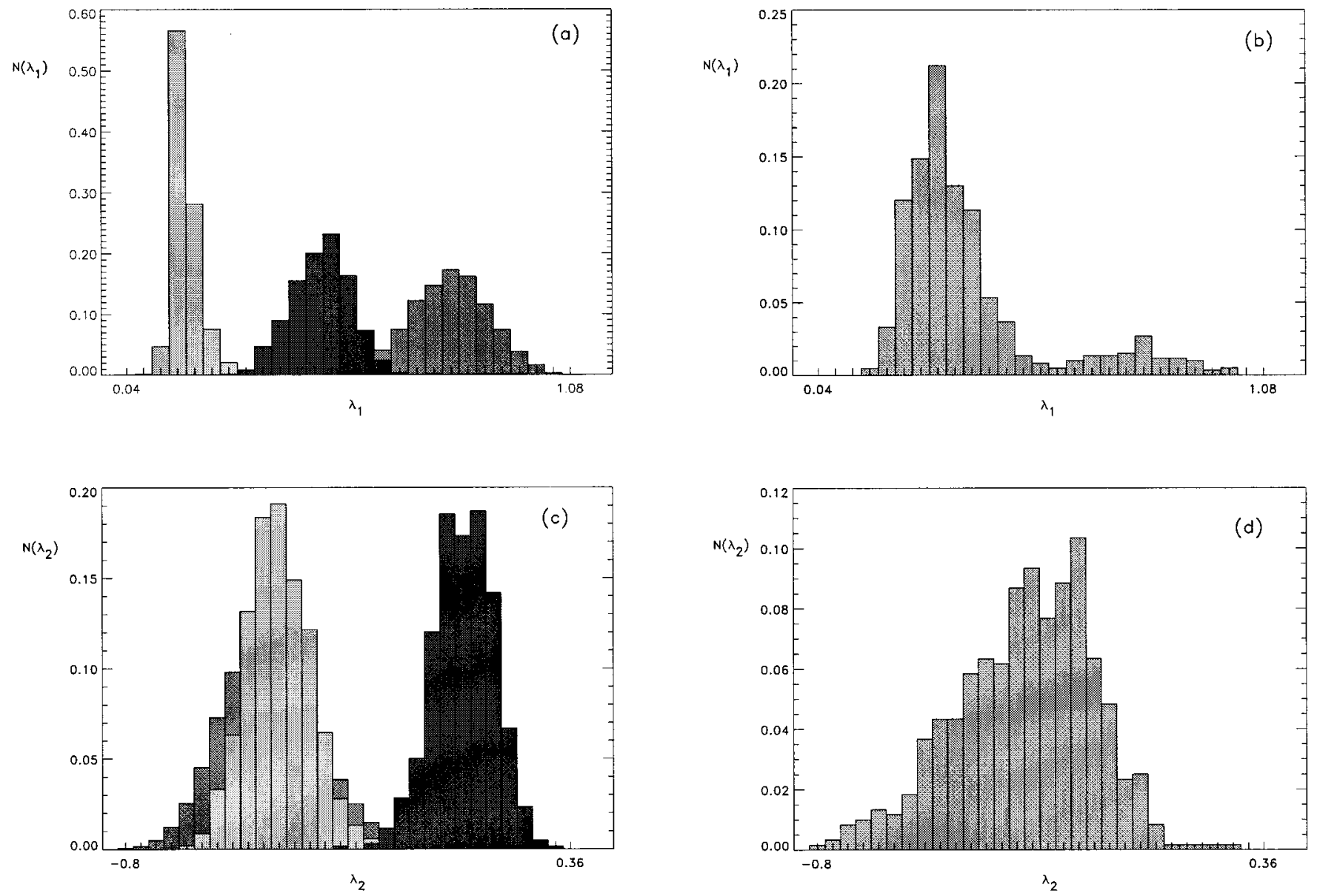

FIG. 10. Normalized histograms of the first, $\lambda_{1}\left[(\mathrm{a})\right.$ and (b)], and second, $\lambda_{2}[(\mathrm{c})$ and (d)], finite-time Lyapunov exponents of the saddles in Fig. 9. The lightest columns in (a) and (c) correspond to the horseshoe saddle in Fig. 9(c), the intermediate ones to the one in Fig. 9(a), and the darkest ones to the Hénon saddle in Fig. 9(b). The histograms in (b) and (d) correspond to the nonattracting invariant set of Fig. 9(d). All histograms are normalized [i.e., $N(\lambda)$ is the fraction of trajectories with that value of $\lambda$ ], and have been done over a total of $N$ trajectories of length 50 with $N=700$ for the Hénon saddle and the postcrisis invariant set, $N=2900$ for the saddle in Fig. 9 (c), and $N=5800$ for the one in Fig. 9(a).

horseshoe saddle suddenly enlarges. After this crisis, using the PIM-triple method, we find a nonattracting chaotic set that contains both 1D and 2D unstable periodic orbits. We show a projection of this set in Fig. 9(d). Comparing Figs. 9(a), 9(b), and 9(d), it looks like the new invariant set "contains" both the horseshoe and the Hénon saddles that existed before the crisis. Actually, we have found that there is another horseshoe saddle that also plays a role in the process. We show a projection of this saddle in Fig. 9(c), at $\rho=5.40$. Our simulations indicate that (a smooth deformation of) all three saddles are contained in the invariant set in Fig. 9(d). Although we cannot prove this, we have checked numerically that a typical orbit on the postcrisis invariant set comes within a distance that is of the order of $4 \times 10^{-2}$ from certain periodic orbits that belonged to the precrisis saddles. We conjecture that the unstable set of the Hénon saddle intersects the stable set of the horseshoe saddle of Fig. 9(a), at $\rho \geqslant 5.28$, because typical trajectories, that start close to the Hénon saddle, spend a long time close to the horseshoe one before they eventually land on the attractor of the system (which, for $\rho=5.28$, is a period 2 orbit). The simulations suggest that, at some parameter value, $\rho^{* *}$, $5.28<\rho^{* *}<5.49$, the horsehoe saddles of Figs. 9(a) and 9(c) form one (indecomposable) invariant set and that, after this happens, the 1D unstable manifold of some of the periodic orbits in this set intersects the stable set of the Hénon saddle. Although we cannot follow numerically all the sequence of events that takes place for $5.28<\rho<5.49$, we can conclude that, at $\rho \geqslant 5.49$ (i.e., after the crisis), there is an invariant set that contains both 1D and 2D unstable periodic orbits. This is reflected in the finite-time Lyapunov exponents, whose distributions we show in Fig. 10. Figure 10(a) contains histograms for the first finite-time exponents of the horseshoe saddle at $\rho=5.28$, the other horseshoe saddle at $\rho=5.40$ (light grey), and the Hénon saddles at $\rho=5.28$ (dark grey). Figure 10(b) contains a similar histogram, but for the postcrisis invariant set (at $\rho=5.49$ ). Figures $10(\mathrm{c})$ and $10(\mathrm{~d})$ are similar to Figs. 10(a) and 10(b), but for the second Lyapunov exponent. There, we observe that the distribution of the postcrisis second Lyapunov exponent is spread over both positive and negative values. This is an indication that there are regions where there are two expanding directions (which are close to the 2D unstable periodic orbits), while there are others where there is only one (which are close to the 1D unstable periodic orbits). Furthermore, we have found several $1 \mathrm{D}$ and $2 \mathrm{D}$ unstable periodic orbits that, within numeri- 
cally accuracy, seemed to belong to the invariant set of Fig. 10(d). On the other hand, both postcrisis distributions look like a composition of the previous ones, as those encountered in other types of crises [31]. This reinforces the idea that the postcrisis invariant set contains the horseshoe and the Hénon saddles. We think that this is the reason why there are 1D and 2D unstable periodic orbits inside the same saddle, at $\rho=5.49$. Furthermore, using the formulas obtained in [32] to estimate the dimension of the horseshoe saddle, $D_{h}$, at $\rho=5.43$ (when both horseshoe saddles seem to be part of the same invariant set) and of the Hénon stable set, $D_{s}$, we obtain $D_{h} \approx 1.997$ and $D_{s} \approx 3.5$. Thus, we expect them to intersect, generically, in the 4D phase space of the doublerotor map.

We could follow the invariant set (with 1D and 2D unstable periodic orbits) up to $\rho \approx 6.85$. At this parameter value, the attractor of the map suffers a crisis, after which it increases significantly in size. Our numerical calculations indicate that the invariant set we followed is inside the attractor after this crisis. Furthermore, as shown in [2], after the crisis, the second finite-time Lyapunov exponent of the attractor is spread over both positive and negative values. Also in this case, we made pictures, equivalent to those in Fig. 10, obtaining strikingly similar results. We then conclude that it is the presence of the Hénon saddle that is ultimately responsible for the coexistence of $1 \mathrm{D}$ and $2 \mathrm{D}$ unstable periodic orbits inside the same chaotic attractor.

\section{CONCLUSIONS}

We have studied various types of nonattracting chaotic sets in maps of more than 2 dimensions, maps that have not been studied very often in the physics literature. We have shown that, in this kind of maps, both chaotic saddles of horseshoe type and Hénon saddles can exist. Hénon saddles are nonattracting chaotic sets that are Hénon attractors when the dynamics is restricted to an invariant manifold of lower dimension than the phase space. This means that they are smooth along one unstable direction, while chaotic saddles of horseshoe type are not (they are products of Cantor sets or products of Cantor sets and isolated points). Typical trajectories on Hénon saddles have at least two positive Lyapunov exponents, while there are horseshoe saddles with only one positive Lyapunov exponent.

We have used the PIM-triple method to obtain numerical trajectories on both types of chaotic saddles. Although the PIM-triple method was originally developed for saddles of horsehoe type with only one unstable direction, we have shown, in this paper, that it can also be used for Hénon saddles such that the strongest unstable direction is transverse to the manifold on which the saddle is attracting. In fact, we have shown that, using this method, we can follow a whole sequence of bifurcations on this invariant manifold that eventually gives rise to a Hénon saddle. All the examples shown in this paper correspond to the double-rotor map [19], a 4D map that describes the simple mechanical system shown in Fig. 5. As far as we know, this is the first time that a Hénon saddle and all the bifurcations that lead to its formation are obtained numerically in any diffeomorphism. Using the PIM-triple method we have also found saddles of horseshoe type with one unstable direction, in the double-rotor map.

Invariant sets that are not attracting have important effects on the observable dynamics [7]. In particular, Hénon saddles can give rise to the existence of invariant sets that contain periodic orbits with different numbers of expanding directions. Moreover, these invariant sets can exist for a whole range of parameter values, i.e., they persist. We have shown an example of how an invariant set of this type can exist, due to the presence of a Hénon saddle, for the double-rotor map. In the example, there is a crisis at which the unstable manifold of a periodic orbit, that belongs to a horseshoe saddle, intersects the stable set of the Hénon saddle. After this crisis, a new invariant set, containing periodic orbits with one and two expanding directions, exists. This becomes evident when the distribution of the precrisis and postcrisis finite-time Lyapunov exponents is studied. The distribution of the second Lyapunov exponent is spread over both positive and negative values after the crisis. It is interesting to note that this invariant set is incorporated into the attractor at a larger parameter value. After this, the distribution of the second finite-time Lyapunov exponent of a typical trajectory on the attractor is also spread over positive and negative values.

In fact, as mentioned in [2], typical trajectories that visit the vicinity of periodic orbits with different numbers of expanding directions have finite-time Lyapunov exponents that change sign along the orbit. If this causes the exponent to fluctuate about zero, then any noise added to the trajectory (such as the one that is unavoidable in numerical simulations) makes the noisy trajectory basically unshadowable [1]. For this reason, numerically generated trajectories of this type are highly unreliable. On the other hand, if the noise is intrinsic, then the meaning of the model is at stake. These behaviors can be due to the presence of a Hénon saddle. Furthermore, Hénon saddles are generically formed at certain types of homoclinic bifurcations in diffeomophisms of more than two dimensions [21]. These reasons show the importance of studying higher-dimensional maps, Hénon saddles, and the new type of dynamical behaviors they can give rise to.

\section{ACKNOWLEDGMENTS}

This work was supported by the University of Buenos Aires, CONICET, and Fundación Antorchas. We acknowledge useful conversations with Gabriel Mindlin.

\section{APPENDIX}

The PIM-triple procedure for finding nonattracting invariant sets, $\mathcal{S}$, and, in particular, chaotic saddles, can be divided into two steps [23]. The first one (the static problem) consists of finding a segment, $I_{0}$, that is as small as we want and that is as close as we want (always within numerical accuracy) to a point in the stable set of $\mathcal{S}, W^{S}(\mathcal{S})$. In order to do this, we first choose a subset of phase space, $\mathcal{R}$, called the restraining region, which we think contains the invariant nonattracting set, $\mathcal{S}$, but no attractors. $\mathcal{R}$ can be multiply connected, but there are always open sets entirely contained in it. Thus, $\mathcal{R}$ also contains smooth subsets of $W^{s}(\mathcal{S})$. For example, suppose that we want to obtain the invariant set of the horseshoe 
map in the unit square. $\mathcal{R}$ can either be the unit square or it can be two horizontal or two vertical stripes in which the invariant set is entirely contained (see, e.g., [12], p. 230). In either case, a set of (horizontal) segments contained in the stable manifold of $\mathcal{S}$ will be inside $\mathcal{R}$. After $\mathcal{R}$ is defined, we choose a segment, $I_{0}^{\prime} \subset \mathcal{R}$, which, for the method to work, must intersect $W^{S}(\mathcal{S})$ transversally. Now, this will occur generically if the dimension of $W^{s}(\mathcal{S})$ is big enough, in particular, if $\operatorname{dim}\left(W^{s}(\mathcal{S})\right)+1-m \geqslant 0$, where $m$ is the phase space dimension. So, by trial and error, we will generically find such an intersection, provided that $\operatorname{dim}\left(W^{s}(\mathcal{S})\right) \geqslant m-1$. This will occur, for example, in the case of the horseshoe map already mentioned, since $m=2$ and $W^{s}(\mathcal{S})$ is a Cantor set of 1D manifolds (horizontal lines). The same will happen for any value of the phase space dimension, $m$, if $W^{s}(\mathcal{S})$ is the union of $(m-1)$-dimensional manifolds, as in the case of a horseshoe-type invariant set with only one unstable direction. Now, in order to find an arbitrarily small segment $I_{0} \subset I_{0}^{\prime}$ that intersects $W^{s}(\mathcal{S})$, the method works under the assumption that the intersection consists of disconnected points (it has been proved in [23] that it converges in this case). In particular, it works for the horseshoe map since, in that case, the intersection will be a Cantor set [unless the segment is exactly horizontal and is contained in $W^{s}(\mathcal{S})$, but the probability that this occurs in the general case is zero]. Now, Hénon saddles have at least two positive Lyapunov exponents, and thus, at least two expanding directions. Why may the method apply? Let us consider a Hénon saddle, $\mathcal{S}$, in an $m$-dimensional phase space, with two expanding directions. Such a saddle is attracting in an $(m-1)$-dimensional invariant manifold. Therefore, $W^{S}(\mathcal{S})$ will generically intersect transversally a (1D) segment. On the other hand, although the invariant manifold, and thus $W^{s}(\mathcal{S})$, can fold onto itself, there will always be gaps in between foldings, so that the intersection of the manifold with a segment that intersects it transversely can be at most a Cantor set. This means that we can solve the static problem for a Hénon saddle using the PIM-triple method.

Once the static problem is solved, $I_{0}$ is iterated forward in time by the map, $f$. Given that $I_{0}$ is sufficiently small, $f\left(I_{0}\right)$ will be another segment, $I_{1}^{\prime}$, typically longer than $I_{0}$. In the case of only one positive Lyapunov exponent, $I_{1}^{\prime}$ will be better aligned along the expanding direction than $I_{0}$ and will also intersect $W^{s}(\mathcal{N})$ transversally. The method, then, proceeds as in the previous step: a very small segment $I_{1} \subset I_{1}^{\prime}$ is found that also intersects $W^{s}(\mathcal{N})$ transversally. All these steps are repeated so that a sequence of segments $I_{0}$, $I_{1} \subset f\left(I_{0}\right), I_{2} \subset f\left(I_{1}\right) \subset f^{2}\left(I_{0}\right), \cdots, I_{n} \subset f\left(I_{n-1}\right) \subset f^{n}\left(I_{0}\right)$, is obtained, all of which intersect $W^{s}(\mathcal{S})$ transversally. It can be proved that there is a forward orbit, $\left\{x_{i}\right\}_{i \geqslant 0}$ in $W^{S}(\mathcal{S})$, such that the distance between $I_{i}$ and $x_{i}$ is very small, $\forall i \leqslant n$ and very large $n$ [23]. Since an orbit in $W^{S}(\mathcal{S})$ will approach $\mathcal{S}$ as $n \rightarrow \infty$, neglecting the first segments, we get, by this procedure, an approximation of an orbit in $\mathcal{S}$. Furthermore, if the segments are sufficiently small, then a plot of the sequence $I_{0}, I_{1}, \ldots, I_{n}$ would be indistinguishable from the real trajectory made out of points. In the case of the Hénon saddle, since there are two expanding directions, the sequence of segments will tend to align along the most unstable direction. Therefore, the various $I_{i}$ will intersect the invariant manifold on which $\mathcal{S}$ is attracting transversally only if the strongest expanding direction is also transverse to this manifold. Under such condition, the PIM-triple method can be applied to obtain Hénon saddles with two positive Lyapunov exponents. Moreover, the fact that we indeed find an invariant set with two positive Lyapunov exponents by "probing" the space with a segment is in itself proof that its stable set is at least $(m-1)$-dimensional.
[1] S. Dawson, C. Grebogi, T. Sauer, and J.A. Yorke, Phys. Rev. Lett. 73, 1927 (1994).

[2] S. P. Dawson, Phys. Rev. Lett. 76, 4348 (1996).

[3] H.G. Solari, M.A. Natiello, and G.B. Mindlin, Nonlinear Dynamics. A Two Way Trip from Physics to Math (IOP Publishing Co, Bristol, 1996).

[4] R. Devaney, An Introduction to Chaotic Dynamical Systems (Addison-Wesley, New York, 1993).

[5] C. Grebogi et al., Phys. Rev. A 36, 3522 (1987); 37, 1711 (1988).

[6] C. Grebogi et al., Physica 7D, 181 (1983); Phys. Rev. A 36, 5365 (1987).

[7] E. Ott, Chaos in Dynamical Systems (Cambridge University Press, Canada, 1993), p. 151.

[8] T. Tél, in Directions in Chaos, Vol. 3, edited by H. Bai-lin (World Scientific, Singapore, 1990), p. 149; T. Tél, in STATPHYS 19, edited by H. Bai-lin (World Scientific, Singapore, 1996).

[9] D.P. Lathrop and E. Kostelich, Phys. Rev. A 40, 4028 (1989); W. L. Ditto et al., Phys. Rev. Lett. 65, 3211 (1990); G.B. Mindlin et al., J. Nonlinear Sci. 1, 147 (1991).

[10] I.M. Jánosi et al., Phys. Rev. Lett. 73, 529 (1994); I. Jánosi and T. Tél, Phys. Rev. E 49, 2756 (1994).

[11] S. Smale, Bull. Am. Math. Soc. 73, 747 (1967).

[12] J. Guckenheimer and P. Holmes, Nonlinear Oscillations, Dynamical Systems and Bifurcations of Vector Fields (Springer, New York, 1986).

[13] There is a large body of work done concerning extended (i.e., infinite dimensional) systems. In particular, there are many works that try to get an insight into this type of systems by analyzing coupled map lattices. When we say that the most widely studied maps are those of one or two dimensions, we refer to those of low enough dimension that are used as prototypical examples of various dynamical behaviors and that have helped develop the theory of dynamical systems. Lately, there has also been some interest in systems with a number of dimensions slightly higher than two in connection with the subject of riddled basins [14]. However, in the examples analyzed in this case there is usually some kind of symmetry that we do not need for our studies.

[14] E. Ott et al., Phys. Rev. Lett. 71, 4134 (1993); E. Ott and J. Sommerer, Phys. Lett. A 188, 39 (1994).

[15] Here we use the poorly defined concept of an attractor being "smooth", along the unstable directions so as to avoid getting 
into very deep mathematical ideas whose full discussion goes beyond the scope of this paper. The attractor being smooth means that there is an invariant probability measure that has absolutely continuous conditional measures on unstable manifolds, i.e., an SBR measure. For more details about what this means we refer the reader to [17]. Actually, the existence of an SBR measure for Hénon maps has only been proved for certain regions of parameter values by Benedicks and Young in [16], building upon the work of M. Benedicks and L. Carleson, Ann. Math. 133, 73 (1991).

[16] M. Benedicks and L.-S. Young, Inv. Math. 112, 541 (1993).

[17] L.-S. Young, in From Topology to Computation: Proceedings of the Smalefest, edited by M.W. Hirsch et al., Ergodic Theory of Dynamical Systems (Springer, New York, 1993), p. 201.

[18] H.E. Nusse and J.A. Yorke, Dynamics. Numerical Explorations (Springer, New York, 1994).

[19] C. Grebogi, E. Kostelich, E. Ott, and J.A. Yorke, Physica D 25, 347 (1987).

[20] R. Romeiras, C. Grebogi, E. Ott, and W.P. Dayawansa, Physica D 58, 165 (1992).

[21] N. Romero, Ergod. Theor. Dynam. Sys. 15, 735 (1995).

[22] L. Mora and M. Viana, Acta Mathematica 171, 1 (1993).

[23] H.E. Nusse and J.A. Yorke, Physica D 36, 137 (1989); Ergod. Theor. Dynam. Sys. 11, 189 (1991); H.E. Nusse and J.A. Yorke, Nonlinearity 4, 1183 (1991).

[24] G. Nicolis and V. Balakrishnan, Phys. Rev. A 46, 3569 (1992); P. Peters and G. Nicolis, Physica (Amsterdam) 188A, 426 (1992); X. Wu and R. Kapral, Phys. Rev. Lett. 70, 1940 (1993)
[25] Actually, the mathematical model is an idealization of the real system and usually not all points in the phase space correspond to states that can be physically achieved.

[26] A periodic point is stable if for every $\epsilon$ neighborhood, $U_{\epsilon}(\bar{x})$, there is a $\delta$ neighborhood, $U_{\delta}(\bar{x})$, such that if $x$ $\in U_{\delta}(\bar{x})$, then $f^{n}(x) \in U_{\epsilon}(\bar{x})$ for all $n \geqslant 0$. Otherwise, it is unstable.

[27] R. Mañe, Ergodic Theory of Differentiable Dynamics (Springer, Berlin, 1987).

[28] V.I. Oseledec, Trans. Moscow Math. Soc. 19, 197 (1968).

[29] Both the concepts of "almost every point" and "typical point" have a meaning in terms of an invariant measure. In particular, the result about typical orbits having the same Lyapunov exponents holds for ergodic invariant measures. For more details see [27].

[30] Actually basins, and in particular basin boundaries, can also undergo dramatic changes at certain parameter values as described in C. Grebogi, E. Ott, and J.A. Yorke, Phys. Rev. Lett. 56, 1011 (1986); Physica D 24, 243 (1987). Although we cannot guarantee what happens with the whole basin between $\rho_{1}$ and $\rho_{2}$, we can say that at least a neighborhood of $\mathcal{A}_{2}$ which lies inside $B_{2}$ is a smooth deformation of a neighborhood of $\mathcal{A}_{1}$ in $B_{1}$. In many cases this is not just a small neighborhood, but the bulk of the basins.

[31] K.G. Szabó and T. Tél, Phys. Lett. A 196, 173 (1994).

[32] B. R. Hunt, E. Ott, and J.A. Yorke, Phys. Rev. E 54, 4819 (1996). 